Reseach Paper

\title{
Cause and Effect Relationship between the Dynamics of Accelerator and Brake Pedals during Emergency Braking
}

\author{
Albert Podusenko ${ }^{1)}$ Vsevolod Nikulin ${ }^{1)}$ Ivan Tanev ${ }^{1)}$ Katsunori Shimohara ${ }^{1)}$ \\ 1) Doshisha University, Graduate School of Science and Engineering \\ 1-3 Tatara-miyakodani, Kyotanabe, Kyoto 610-0321, Japan (E-mail: podusenko2016@sil.doshisha.ac.jp)
}

Received on November 15, 2017

\begin{abstract}
We explore a feasibility of reducing the time lag between the instant when a driver decides to apply brakes and the instant the car reaches its maximum deceleration in emergency braking situations. The former event is manifested by the energetic lifting of the accelerator pedal, while the latter - by pressing the brake pedal to its maximum position. The time lag between these two events is a personal trait, and, in most cases, it is about few hundreds milliseconds. Depending on the speed, the considered time lag corresponds to several meters of distance traveled by car. By reducing this time lag, we intend to reduce the overall braking distance of the car in emergency braking. As the first step in our research towards the reduction of the time lag we consider investigating the cause and effect relationship (if any) between the event of energetic lifting the accelerator pedal and pressing the brake pedal to its maximum position. Presence of such a relationship would indicate that, in principle, the imminent braking could be predicted from the movement of the accelerator pedal, and consequently - an automated proactive braking could be applied well before the driver would have been able to apply the brakes.
\end{abstract}

KEY WORDS: Safety, cause and effect relationship, emergency braking, time lag, proactive braking [C1]

\section{Introduction}

Despite the diversity of the existing driving aids ${ }^{(1)}$, that are aimed at preventing the traffic accidents, the development of new ones remains a very important aspect of improvement of safety of the road traffic. Braking assistance is one of the most important types of driving aids.

Nowadays the implementation of fully automated braking mechanisms faces several challenges which are related to both to the reliability and validity of sensory information and to adequate consideration of various human-related factors (e.g., risk homeostasis theory ${ }^{(2)}$ argues that fully automated driving aids might be detrimental to the road safety because they tend to create an inflated sense of safety in drivers). Therefore, instead of considering the fully automated braking system, we focus on automated brake assistance to the human drivers.

The purpose of our work is investigating the feasibility of predicting the emergency braking situation from the way the driver lifts the accelerator pedal. For a better understanding of the dynamics of the accelerator pedal, we obtained the time series of the typical motion patterns of both the accelerator and brake pedals in various driving situations - such as accelerating, cruising, slowing down, normal braking and emergency braking in experiments with a full-scale drive simulator ${ }^{(3)}$. Snapshot of the driving simulator is shown in Figure 1. The typical dynamics of both the accelerator and brake is illustrated in Figure 2.

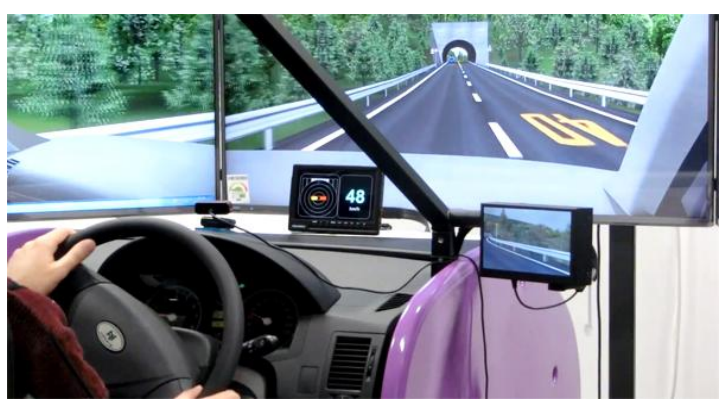

Fig. 1. Snapshot of the full-scale drive simulator used in our research.

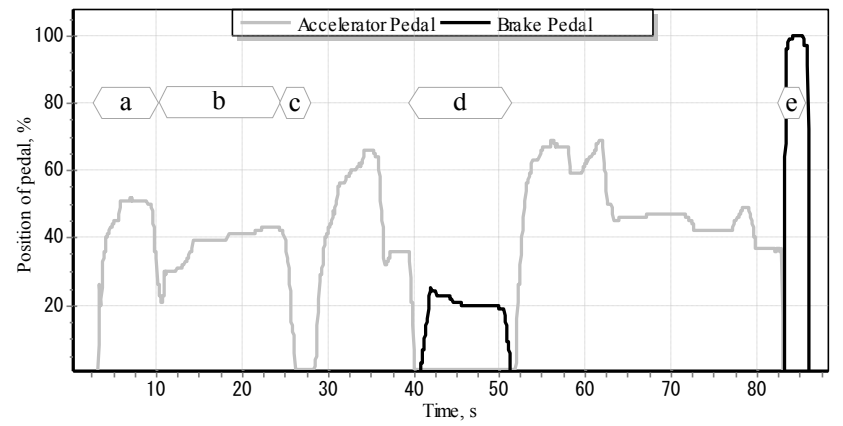

Fig. 2. Typical dynamics of accelerator- and brake pedals during accelerating (a), cruising (b), slowing down (e.g., approaching corner, c), normal braking (e.g., approaching a stop sign, d) and emergency braking (e), respectively.

The closer scrutiny of the typical dynamics of pedals during emergency braking situation (as shown in Figure 3) suggest that it 
might be possible to predict that the driver is about to press the brake pedal (and consequently - to classify the emergency braking situation) from the dynamics of lifting the accelerator pedal by handcrafting a programming code that (i) first calculates the rate of decrease of the position of accelerator and then compares this rate to a predetermined threshold. The correct classification of driver's intention for applying emergency braking will allow us to reduce the time lag between the instant (i) when the driver moves their foot away from the accelerator pedal (Figure 3a) and then (ii) presses the brake pedal to its maximum position (Figure 3c) in emergency braking. The resulting time lag that we intend to reduce is defined as a sum of the intervals (b) and (c).

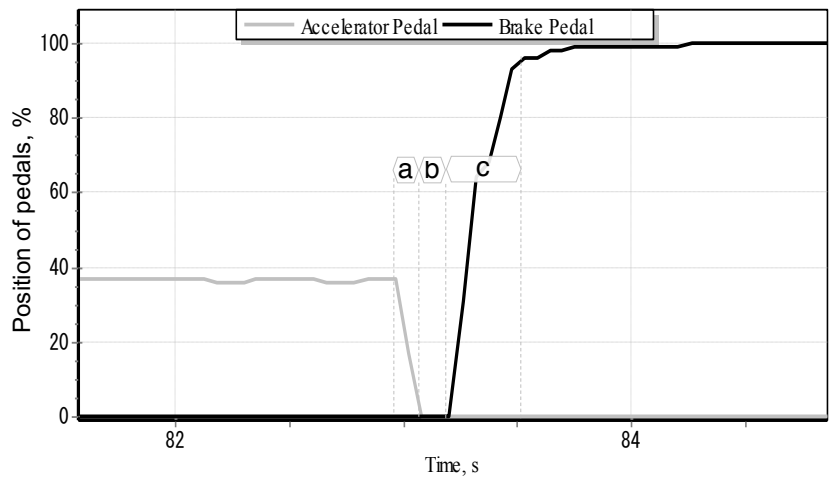

Fig. 3. Typical dynamics of accelerator- and brake pedals during emergency braking. The braking behavior of the driver could be decomposed into the following three actions: lifting the accelerator (a), moving the leg from accelerator to the brake pedal (b), and pressing the brake pedal to its (almost) maximum position. Depending on physical and cognitive condition of the driver, the corresponding time lags of these actions might significantly vary

Based on the above mentioned assumptions we organized our work in the following three main stages: (i) acquiring and analyzing the data (as time series) of the dynamics of the accelerator and brake pedals of the car, (ii) classifying the emergency braking and normal driving situations from the acquired dynamics of pedals, and (iii) verifying the quality of the learned classifier.

\section{Data Acquisition and Analysis}

\subsection{Data Acquisition}

We set up the environment in a full-scale Forum- 8 drive simulator and asked 11 drivers (men and women of the age 22-52) to drive this simulator on different tracks. We conducted two types of experiments with each test driver. The first type of the experiments was related to the normal driving. Test drivers were asked to drive a car in various traffic situations such as entering and driving in busy highway, driving in the countryside, etc. The most relevant request for this type of experiments was to avoid the use of emergency braking. In the second type of experiments, drivers were audibly signaled (at random sections of the test tracks) to stop the car suddenly in a way which imitates the emergency braking situation. The time series data of the accelerator and braking pedal were being recorded by the simulated parametric data recorder with a sampling frequency of $25 \mathrm{~Hz}$. In total, we obtained 929 samples of lifting the accelerator pedal, 383 of them belonging to emergency braking (class " 1 ") and 546 - to normal deceleration or braking (class " 0 ").

\subsection{Analysis}

In order to confirm the significance of the delay between the lifting of accelerator and pressing the brake pedal completely, we analyzed the breakdown of the considered time lag during the recorded 383 emergency braking situations. The breakdown is shown in Figure 4.

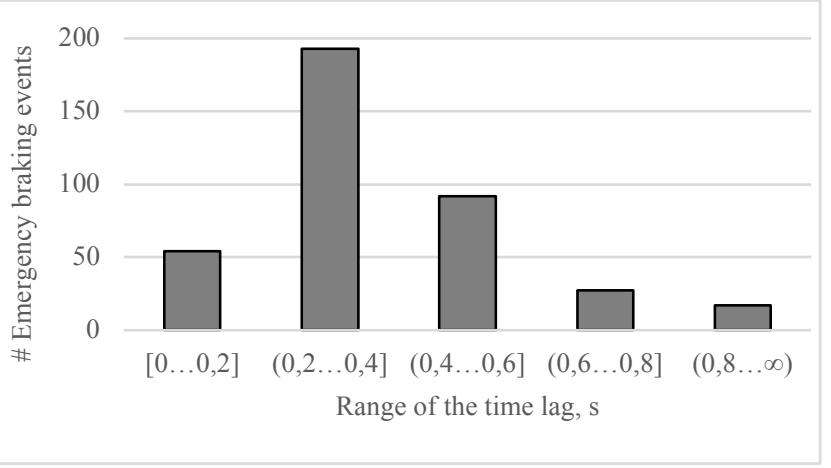

Fig. 4 Breakdown of the time lag between the lifting of accelerator and pressing the brake pedal completely

As shown in Figure 4, in about 50\% (193 of 383) of emergency braking events, the range of time lag is $[0.2 \mathrm{~s} \sim 0.4 \mathrm{~s}$ ] which corresponds to the traveled distance of between $2.2 \mathrm{~m}$ and $4.4 \mathrm{~m}$ for the speed of the car of $40 \mathrm{~km} / \mathrm{h}$. However, for the speed of $70 \mathrm{~km} / \mathrm{h}$, the traveled distance eventually increases to $7.7 \mathrm{~m}$. For longer time lags (pertinent to tired or elderly drivers) this distance would increase even further.

Apart from the dynamics of accelerator and braking pedals we extracted the highest position of the accelerator pedal before starting the deceleration $(\mathrm{mP})$, the maximum and average rate of lifting the accelerator ( $\mathrm{mR}$ and $\mathrm{aR}$ respectively), the maximum and average rate of pressing the brake pedal, and the maximum position of the pressed brake pedal.

Each point (sample) in Figure 5, 6, and 7 corresponds to the registered event during the acquiring the time series of dynamics of pedals. In the onset of our research, we believed that it would be possible to achieve a good accuracy in detecting the emergency braking by considering either $m R$ or $a R$. For instance, if $m R(a R)$ is higher than a certain threshold we could conclude that the event could be classified as emergency braking. This idea was based on assumption that the points of class " 1 " and class " 0 " would always form compact, non-overlapping clusters. Higher rates of pressing the brake pedal correspond to emergency braking situations, and they are often preceded by the energetic release of the accelerator. However, as it can be seen from Figures 5, 6, and 7 , a significant number of the samples which have low rates of releasing the accelerator, and low rates of pressing the brake pedal belong to class " 1 " but could not be easily distinguished from the 
samples belonging to class " 0 ". The reason of these ambiguous regions (gray zones) is that sometimes during the emergency braking situations the drivers start to release accelerator pedal when $\mathrm{mP}$ is low, therefore neither the value of $m R$ nor $a R$ is significantly large

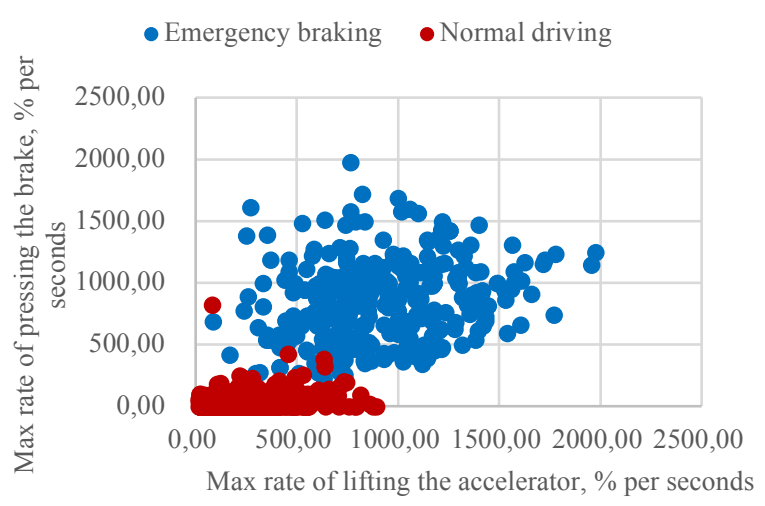

Fig. 5 Relationship between the maximum rate of lifting the accelerator and maximum rate of pressing the brake pedal

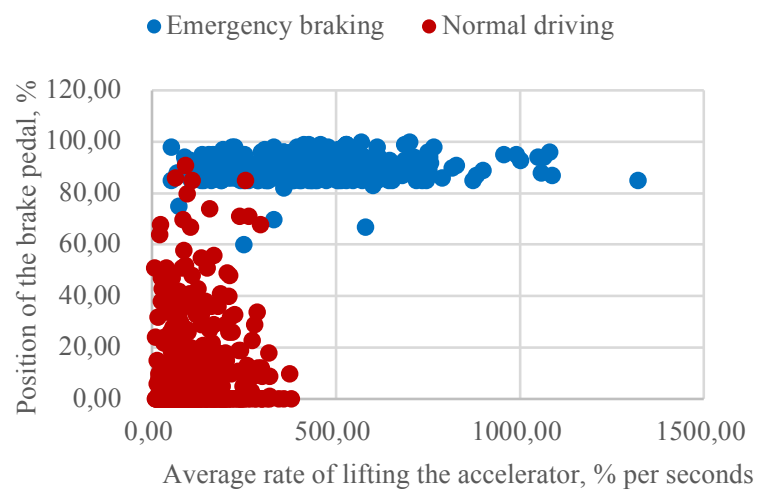

Fig. 6. Relationship between the average rate of lifting the accelerator and the position of the pressed brake pedal.

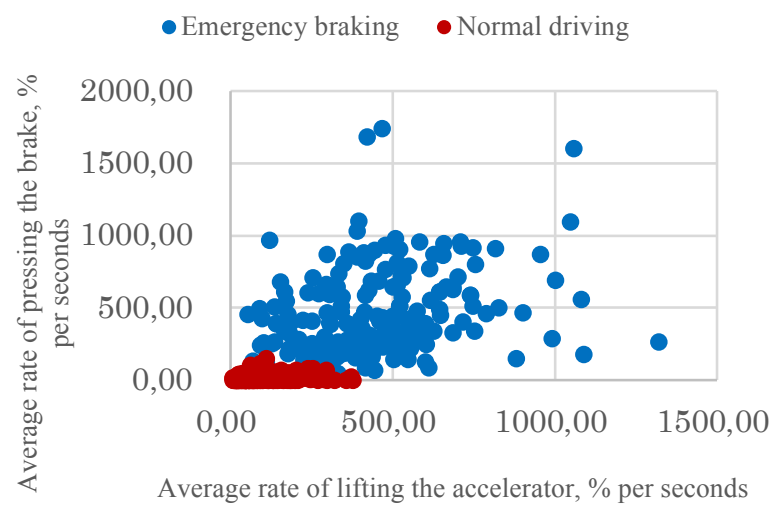

Fig. 7. Relationship between the average rate of lifting the accelerator and the average rate of pressing the brake pedal.
Apparently, no single feature of the pattern of lifting the accelerator could be used as a sole classification criterion. This finding motivated us to explore a machine learning approach such as support vector machine - for automated development of the classifier of emergency braking situations.

\section{Proposed Approach}

\subsection{Support vector machine}

Support vector machine $(\mathrm{SVM})^{(4)}$ is one of the best-known methods of machine learning. This method showed promising results in solving many challenging linear and non-linear problems. SVM performs well on high-dimensional and large datasets.

The fundamental idea of SVM is a construction of hyperplane which splits the training set into the required number of classes. In the case of emergency braking detection, we are searching for the hyperplane which separates the dataset into just two classes: class "1" (emergency braking) and class "0" (normal driving or normal braking), respectively. When hyperplane is built, a testing sample is being put on a plane. Depending on its position relative to the hyperplane, we infer that testing sample belongs either to the class emergency braking or that of normal driving.

Formally the problem can be described as follows: given a training set of $N$ points $\left(x_{i}, y_{i}\right)$, where $x_{i} \in \mathbb{R}^{n}$ and $y_{i} \in \mathbb{R}$ are the $i$ th input and output samples respectively. SVM constructs a classifier which can be represented by the following function:

$$
f(x)=\operatorname{sign}\left[\sum_{i=1}^{N} \alpha_{i} y_{i} K\left(x, x_{k}\right)+b\right]
$$

where $x_{k}$ are support vectors, $\alpha_{i}$ are positive real numbers, while $b$ (bias) is a real number. The kernel $K$ can be used in various forms:

$K\left(x, x_{k}\right)=x_{k}^{T} x$ (linear kernel);

$K\left(x, x_{k}\right)=\left(\gamma x_{k}^{T} x+r\right)^{n}$ (polynomial kernel of degree $\left.n\right)$;

$K\left(x, x_{k}\right)=e^{-\gamma\left\|x-x^{\prime}\right\|^{2}}$ (radial basis function kernel);

$K\left(x, x_{k}\right)=\tanh \left[\gamma x_{k}^{T} x+r\right]$ (sigmoid kernel);

The kernel parameters $\gamma=\frac{1}{2 \sigma^{2}}$ and $r$ are real constants.

The learning of SVM lies in finding such $f(x)$ that minimizes the following objective:

$$
O b j=C \sum_{i}^{N} l\left(f\left(x_{i}\right), y_{i}\right)+\Omega\left(\alpha_{i}, b\right)
$$

where the first term $l$ is a loss function, $l(\hat{y}, y)=0$, if $\hat{y}=y$, and the second term $\Omega$ is a penalty function of our model parameters. The parameter $C$ reflects our intention to avoid the misclassification each training sample. For instance, large values of $\mathrm{C}$ would affect the small margin hyperplane which will possibly do fewer errors on the training set. On the other hand, low values of $\mathrm{C}$ will affect the bigger margin hyperplane as shown in Figure 8. 

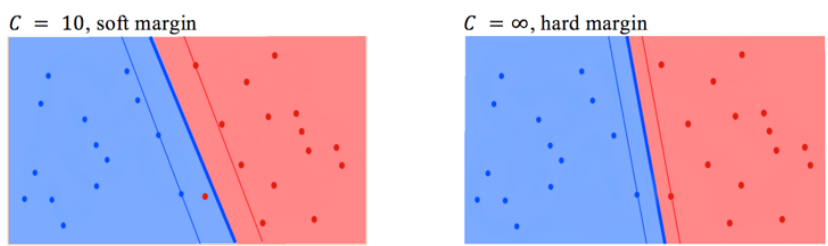

Fig. 8. Example of the influence of the value of parameter $C$ in a sample two-dimensional classification problem.

\subsection{Quality metrics}

In order to evaluate the performance and quality of the trained classifier in full, different metrics must be used. Therefore, in addition to the accuracy which is equal to the percentage of correct answers, we use other metrics like precision, recall, and $f$ score $^{(5)}$. The reason for this is that accuracy doesn't reflect the specifics of the considered problem very well. For example, assuming that the testing set size is 200 , where 60 of samples corresponds to class " 1 " and 140 corresponds to class " 0 " if the classifier detects all samples of class " 0 " the accuracy will be equal to 0.7. However, the classifier would miss all emergency braking events which, in turn, would question the very meaning of using such a classifier.

The aforementioned alternative metrics are more informative. The meaning of these metrics is illustrated in Table 1. The table shows four categories and provides an information about how many times the classifier has given the correct answer and how many times the wrong decision has been adopted.

Table 1. Confusion matrix

\begin{tabular}{|c|c|c|c|}
\hline \multicolumn{2}{|c|}{} & \multicolumn{2}{|c|}{ True condition } \\
\cline { 3 - 4 } \multicolumn{2}{|c|}{} & Positive & Negative \\
\hline \multirow{2}{*}{$\begin{array}{l}\text { Predicted } \\
\text { condition }\end{array}$} & Positive & TP & FP \\
\cline { 2 - 4 } & Negative & FN & TN \\
\hline
\end{tabular}

True positive category (TP) is equal to the number of samples which were classified as class " 1 " (emergency braking) and truly belong to the class "1". False positive (FP) corresponds to the samples for which the correct answer " 0 " (normal driving) but the classifier returns " 1 ". And vice versa, if algorithm classifies the sample as " 0 " but the real answer is " 1 " this sample belongs to false negative (FN). If the sample belongs to the class " 0 " and the classifier returns " 0 " - true negative (TN). Thus, recall and precision can be expressed as follows:

$$
\begin{aligned}
& \text { Precision }=\frac{T P}{T P+F P} \\
& \text { Recall }=\frac{T P}{T P+F N}
\end{aligned}
$$

Both of these metrics describe different aspects of the classifier: higher precision could be achieved with lower FP. Similarly, higher recall corresponds to lower $F N$.

False positives are dangerous in the problem under consideration because false emergency braking could cause a rear-end collision on the road. On the other hand, it is necessary to detect as many cases of emergency brakes as possible. Therefore we should pay attention to both of these metrics and f-score allows us to reflect this intention:

$$
F=2 * \frac{\text { Precision } \times \text { Recall }}{\text { Precision }+ \text { Recall }}
$$

From another perspective, $F$-score could be seen as a harmonic mean of the precision and recall. Factor 2 is introduced to scale the $f$-score up to 1 when both recall and precision are 1 .

\section{Implementation and Experimental Results}

In order to apply the approach of SVM for the emergency brake classification, we use powerful machine learning library for Python programming language - scikit-learn ${ }^{(6)}$. The latter allows a visualization of the dataset in a very representative way. This library provides a number of useful methods and features an $\mathrm{API}^{(7)}$ that adequately supports the adopted learning mechanism.

Before proceeding to the training of the classifier, we separated the dataset into training and testing subsets in the proportion of 7:3. Therefore the testing set included 279 samples, while training set -650 .

\subsection{One-dimensional classification}

Despite the obvious inefficiency of one-dimensional classification, we present the results of this approach with the intention to use its results as a benchmark.

For building a classifier which separates two classes we gradually moved the threshold for both $m R$ and $a R$ before we reached the maximum value of $f$-score as it is shown in Figure 9.

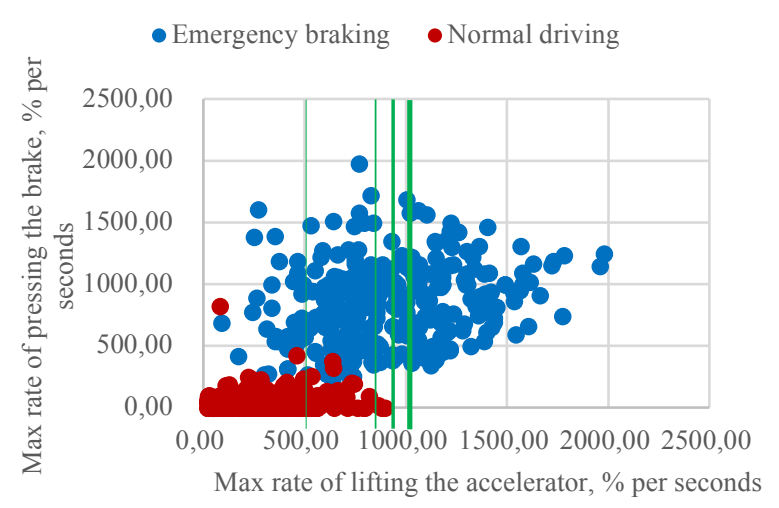

Fig. 9. Example of building one-dimensional classifier.

The best results of the threshold classifier on the testing set are shown in Table 2. It is worth noting that classification with single attribute $a R$ yields a better result than classification with $m R$. 
Table 2. Classification with single attributes $m R$ and $a R$, respectively

\begin{tabular}{|l|c|c|}
\hline \multirow{2}{*}{$\begin{array}{c}\text { Evaluation } \\
\text { metric }\end{array}$} & \multicolumn{2}{|c|}{ Classification method } \\
\cline { 2 - 3 } & With single $m R$ & With single $a R$ \\
\hline TP & 80 & 85 \\
\hline TN & 160 & 162 \\
\hline FP & 10 & 8 \\
\hline FN & 29 & 24 \\
\hline Accuracy & 0.86 & 0.885 \\
\hline Presision & 0.889 & 0.914 \\
\hline Recall & 0.734 & 0.78 \\
\hline F-score & 0.804 & 0.842 \\
\hline
\end{tabular}

\subsection{Multidimensional classification}

In order to prove the potential of improving the quality of classification, we use SVM with RBF kernel and different combination of features. The experimental results of applying SVM classifier for the testing set are shown in Figure 10, Figure 11, Figure 12, Figure 13 and Table 3 below. The results indicate that using more sophisticated classifiers than threshold classifier contributes to the achievement of better results of accuracy, precision, recall, and $f$-score.

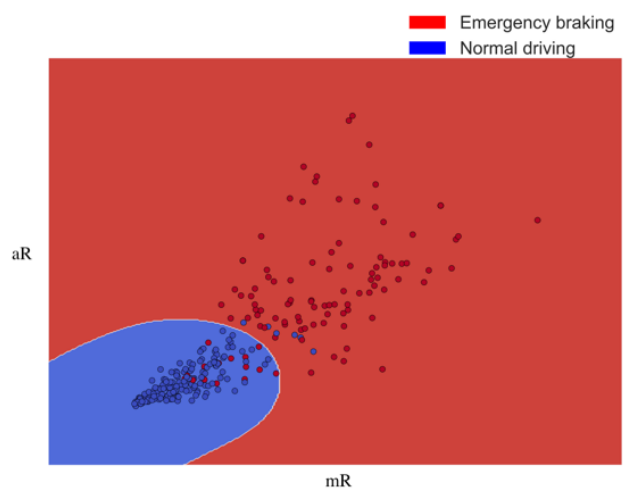

Fig. 10. Visualization of SVM on $m R$ and $a R$.

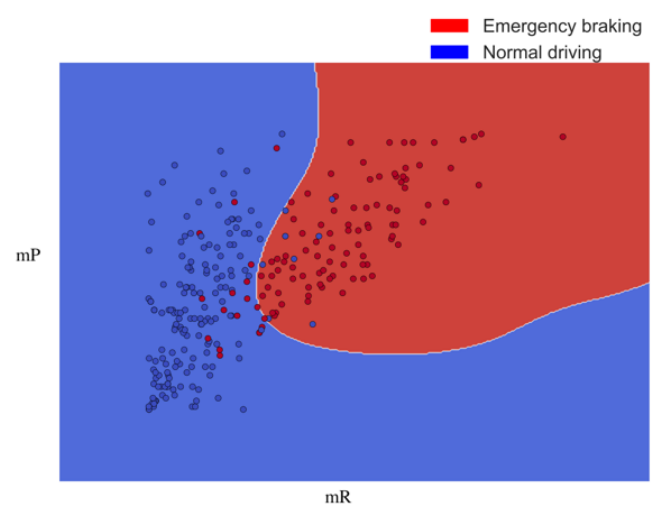

Fig. 11. Visualization of SVM on $m P$ and $m R$.

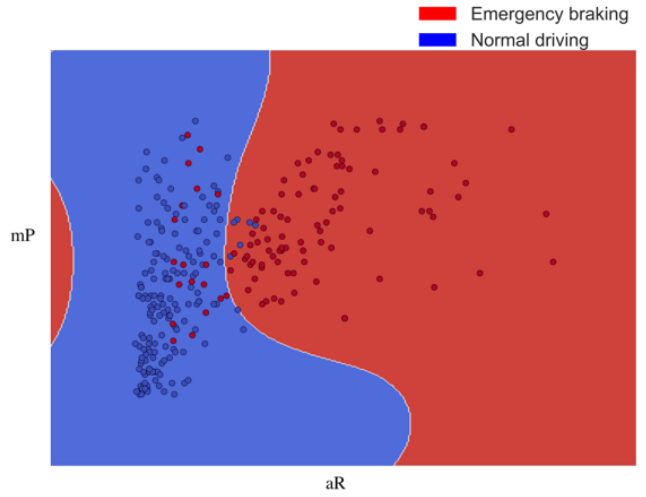

Fig. 12. Visualization of SVM on $a R$ and $m P$.

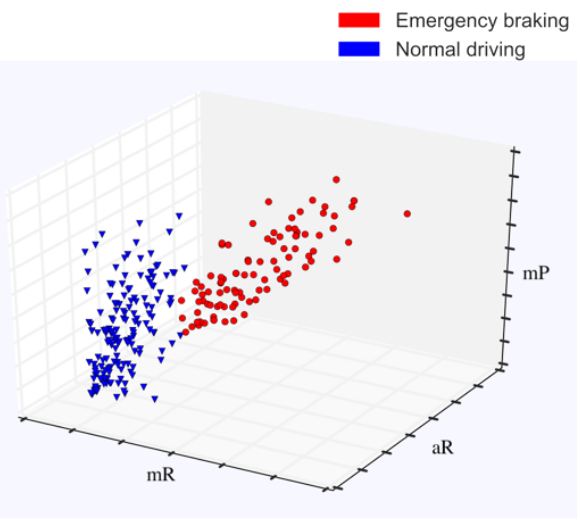

Fig. 13. Visualization of SVM on $a R, m R$ and $m P$

Table 3. Performance of SVM on various combinations of attributes

\begin{tabular}{|l|c|c|c|c|}
\hline \multirow{2}{*}{$\begin{array}{c}\text { Evaluation } \\
\text { metric }\end{array}$} & \multicolumn{4}{|c|}{ Attributes used by the classifier } \\
\cline { 2 - 5 } & $m R$ and $a R$ & $m P$ and $m R$ & $a R$ and $m P$ & $a R, m R$ and $m P$ \\
\hline TP & 94 & 91 & 89 & 95 \\
\hline TN & 164 & 162 & 164 & 164 \\
\hline FP & 6 & 8 & 6 & 6 \\
\hline FN & 15 & 18 & 20 & 14 \\
\hline Accuracy & 0.925 & 0.907 & 0.907 & 0.928 \\
\hline Presision & 0.94 & 0.919 & 0.937 & 0.94 \\
\hline Recall & 0.862 & 0.835 & 0.816 & 0.871 \\
\hline F-score & 0.899 & 0.875 & 0.872 & 0.904 \\
\hline
\end{tabular}

The experimental results indicate the most informative combination of features for SVM are $m R$ and $a R$. It is important to notice that the use of all extracted features increases the values of quality metrics. Despite the fact that we couldn't significantly reduce the number of $\mathrm{FP}$, the number of $\mathrm{TP}$ increased.

\section{Discussions}

In real-world braking situations, the obtained results cannot be considered as a reliable basement for the proposed automated braking system. Technically the precision value of 0.94 means, that the system will wrongly activate the brakes three times out of fifty, while according to recall value, the proposed system will 
miss about $13 \%$ of real emergency braking cases. However, despite the fact that our research was aimed at demonstrating the cause and effect relationship between the dynamics of accelerator and brake pedals during emergency braking, we could obtain the classifier that performs significantly better than one-dimensional classifier. This classifier was integrated into the prototype braking systems and successfully tested on Forum8 driving simulator ${ }^{(8)}$.

The possible drawback of the built classifier is its inability to generalize the data of all drivers who participated in the experiments. It means that, consonant with the concept of "end of average" of the human factors ${ }^{(9)}$, there might be no (generic) classifier that would be good enough for all human drivers. Therefore, in the near future we are planning to investigate the adaptation of generic classifiers to the specific drivers in a real time by applying online learning methodologies.

\section{Conclusion}

We investigated the cause-effect relationship between the patterns of accelerator and brake pedals of road vehicles during emergency braking situations. We confirmed such a relationship by proving the possibility of classifying the emergency braking situations entirely from the motion pattern of the accelerator pedal. Moreover, we demonstrated the necessity of machine learning approaches and used SVM for such a classification. The experimental results suggest that the SVM classifier detects the emergency braking situations with the f-score of 0.904 on the testing set.

However we couldn't detect all TP and TN which might be a result of several factors such as imperfection of the selected classifier for this particular problem, sub-optimal parameters of the classifier, ineffective extraction of features or even the presence of noisy data both in testing and training set.

In our future work we are planning to address these possible drawbacks of the current approach. We should also consider alternative machine learning approaches (e.g., evolutionary computing) of classifying the emergency braking situations. Also, we plan to use cross-validation in order to prevent the overfitting. Our ultimate objective in the near future is the implementation of brake assistant that would be a best match to the driving style of the particular driver.

\section{This paper is written based on a proceeding presented at JSAE FAST-zero' 17 Meeting.}

\section{Acknowledgement}

This work was funded in part by MEXT-supported Program for Strategic Research Foundation at Private Universities in Japan (2014-2018).

\section{References}

(1) J. Laukkonen, 13 Advanced Driver Assistance Systems, URL: https://www.lifewire.com/advanced-driver-assistancesystems-534859 (Accessed 2018.11.04)
(2) G. J. S. Wilde, The theory of risk homeostasis: Implications for safety and health, Risk Analysis, 2, pp.209-225, (1982).

(3) Forum-8 Drive Simulator,

URL: http://www.forum8.co.jp/english/uc-win/road-drivee.htm (Accessed 2018.11.04)

(4) J. Suykens, J. Vandewalle, Least Squares Support Vector Machine Classifiers, Neural Processing Letters 9: 293. doi:10.1023/A:1018628609742, (1999)

(5) T. Fawcett, An Introduction to ROC Analysis, Pattern Recognition Letters. Vol.27, No.8, p.861-874 doi:10.1016/j.patrec.2005.10.010. (2006).

(6) F. Pedregosa et al, Scikit-learn: Machine Learning in Python, JMLR 12, pp. 2825-2830, (2011).

(7) L. Buitinck et al, API design for machine learning software: experiences from the scikit-learn project, (2013).

(8) I. Tanev, A. Podusenko, V. Nikulin, K. Shimohara, Braking Assistance Prototype

URL: $\quad$ https://www.youtube.com/watch?v=Iif46Yqyk1A (Accessed 2018.11.04)

(9) T. Rose, The end of average: How We Succeed in a World That Values Sameness, p.256 (2016). 